\title{
Fire in the Forest: Adverse Effects of Antibiotics on the Healthy Human Gut Microbiome
}

\author{
Manosha Perera ${ }^{1 *}$, Irosha Perera ${ }^{2}$ \\ ${ }^{1}$ School of Dentistry and Oral Health, Griffith University, Queensland, Australia \\ ${ }^{2}$ Preventive Oral Health Unit, The National Dental Hospital (Teaching), Colombo, Sri Lanka
}

Corresponding Author: Manosha Perera, School of Dentistry and Oral Health, Griffith University, Queensland, Australia. Tel: +9471-3271945, Email: manosha.perera@griffithuni.edu.au

Received December 5, 2017; Accepted February 18, 2018; Online Published March 20, 2018

\begin{abstract}
Antimicrobial resistance, especially the emergence of multidrug-resistant human pathogens, remains a serious public health crisis across the globe. The human gut microbiome regulates essential human functions including digestion, energy metabolism, brain function, and immunity by modulating multiple endocrine, neural, and immune pathways of the host. Increasing evidence shows adverse effects of antibiotics on the community structure and functions of healthy gut microbiomes. Short-term antibiotic treatment is able to change the richness and diversity of species into a long-term dysbiotic state. The colonization of invading pathogens is encouraged because of decreased competitive exclusion. Furthermore, the accumulation of antimicrobial resistant genes in the gut microbiome (gut resistome) facilitates the emergence of multidrug-resistant nosocomial pathogens. In this study, the adverse effects of antibiotics on the gut microbiome are highlighted in terms of dysbiosis and the accumulation of resistance genes. In light of evidence of such adverse impacts, several epidemiological studies have been conducted on traditional culture techniques and 16S rRNA metagenomics to assess the compositional and functional changes occurring in the gut microbiome after exposure to antibiotics. They have failed to agree on specific antibioticassociated microbiome and its functional redundancy subsequent to exposure to antibiotics. This mini-review describes the composition and role of a healthy microbiome to understand and appraise the value of gut microbiome and summarizes the current understanding of adverse effects of antibiotics on it.

Keywords: Gastrointestinal Microbiome, Anti-bacterial Agents, Health

Citation: Perera M, Perera I. Fire in the forest: adverse effects of antibiotics on the healthy human gut microbiome. Int J Med Rev. 2018;5(1):19-26. doi:10.29252/IJMR-050104.
\end{abstract}

\section{Introduction}

"Human microbiome" is a term used to define all microorganisms, their genomes (metagenome), and the ecosystems they inhabit on and within the human (Homo sapiens) body. ${ }^{1-}$ 3 Thus, "Super organism" or "Holobiont" are terms currently being used to refer to human and symbiont microbial cells. ${ }^{4}$ In this "super-organism", trillions of bacteria live in various habitats on and within the body, outnumbering eukaryotic cells 10 to 1 , and commencing from the neonatal period. The human body is divided into 5 main microhabitats, namely nasal, oral, skin, gastro-intestinal, and urogenital., Each microhabitat demonstrates variations in microbial composition $^{7}$ by reflecting the uniqueness of a specific ecosystem. Distinct atmospheric and nutritional compositions of a specific ecosystem offer an ideal platform for symbiotic interactions between the various microbes within that ecosystem and the host. ${ }^{6}$ It has been found that compositions of microbiomes from the same location of the body among different individuals are more similar than microbiomes from different locations on the same individual. ${ }^{5}$

The human gastrointestinal (GI) tract is considered to be one of the largest interfaces (250-400 $\left.\mathrm{m}^{2}\right)$ in the body. It harbors a diverse and dynamic microbial population collectively termed the gut microbiome. ${ }^{8}$ These microbes have co-evolved with the host over thousands of years to establish complex and mutually beneficial associations. ${ }^{8,9}$ The gut microbiota participate in numerous physiological functions in the human, which, in turn, regulate immune and metabolic homeostasis in the host. ${ }^{10}$

The widespread use of antibiotics in the past 80 years has killed an incalculable number of microbes, both pathogenicand commensal. ${ }^{11}$ Furthermore, the use of antibiotics significantly alters the community structure of the gut microbiome; this process is known as "dysbiosis". A dysbiotic microbiome may not perform vital functions such as the digestion of food, synthesis of vitamins, or protection against pathogens. ${ }^{12}$ Human gut microbiomes act as a reservoir for antimicrobial resistance genes (ARGs), and most ARGs are harbored by commensal strict anaerobes. ${ }^{9}$ The horizontal transfer of ARGs from gut commensals to opportunistic pathogens in the gut may promote the emergence of multidrug-resistant nosocomial pathogens. The rate of discovery of new antibiotics

Copyright $\odot 2018$ The Author(s). This is an open-access article distributed under the terms of the Creative Commons Attribution License (http:// creativecommons.org/licenses/by/4.0), which permits unrestricted use, distribution, and reproduction in any medium, provided the original work is properly cited. 
cannot compensate for the withdrawal of antibiotics. ${ }^{13}$ It has been speculated that by 2050, 10 million deaths per year worldwide will be attributable to antibiotic resistance. ${ }^{14}$ The adverse impacts of antibiotics on the community structure of the gut microbiome were assessed recently by traditional metagenomic profiling. Relatively few transcriptomic, proteomic, and metabolomic studies have been carried out to determine the impact antibiotics have on the metabolic activities of the gut microbiome. ${ }^{15}$ This emerging field of research provides molecular epidemiological evidence for the adverse effects of antibiotics. Unnecessary disruption to the microbiome and the risk of accumulating ARGs can be avoided by rational use of antibiotics.

\section{Community Composition of the Healthy Human Gut Microbiome}

In past decades, the majority of information on human gut microbiota was provided by conventional culture-based methods. Recent developments in high throughput, nextgeneration sequencing (NGS) technologies have paved the way for mining the gut microbiome at exceptional breadth and length. ${ }^{16}$ The $16 \mathrm{~S}$ ribosomal rRNA gene, which is present in all bacteria and archaea, contains nine highly variable regions (V1-V9) and has become the housekeeping gene in the NGS approach as it allows species to be distinguished better. Approximately $76 \%$ of the sequences obtained from an adult male stool sample belonged to novel and uncharacterized species. ${ }^{17}$ Thus, targeting the $16 \mathrm{~S}$ rRNA gene has evolved as a popular approach to overcoming the inherent limitations of culture-based methods. ${ }^{18}$ Analysis of shorter subregions of this gene in greater depth has become the method of choice, ${ }^{19}$ though it has limitations such as introducing errors. ${ }^{18}$ Against this backdrop, whole-genome shotgun metagenomics have emerged as a more sensitive technique for estimating microbial composition and diversity due to the higher resolution and sensitivity it offers. ${ }^{18}$ It is possible to understand microbial interactions and microbial ecology in a better way. ${ }^{9}$ To date, combined data from MetaHit and the Human Microbiome Project have provided the most inclusive analysis of the associated human gut microbial catalog. ${ }^{20,21}$

The gut microbiome consists of bacteria, archaea, viruses (especially bacteriophages), fungi, and protozoa. The diversity of gut microbiome is great and varies across the different sites of the GI tract. Over 1000 bacterial species belonging to 12 different phyla inhabit the human gut. Of them, the majority have been identified as proteobacteria, Firmicutes, actinobacteria, and Bacteroidetes, of which many remain uncultivated. ${ }^{8}$ Among them, Bacteroidetes and Firmicutes are abundant in the healthy gut microbiome. It has been reported that at least 160 species can be found in the gut of any healthy individual. $^{22}$ The metagenome of the gut microbiome has been estimated to be about 3 million genes, which means it is 150 times larger than that of the human genome. ${ }^{23}$ The fast majority of the bacteria that populate the human gut are strict anaerobes. Moreover, several facultative anaerobic bacteria representing the families of Enterobacteriaceae and Enterococcaceae are ubiquitous members of the human gut microbiome at levels that are at least 100-fold lower than those of the strict anaerobe gut commensals. ${ }^{22}$ The presence of country-specific microbial signatures in Chinese and Danish individuals has been revealed in an extensive catalog of gut microbiomes. ${ }^{24}$ Furthermore, another study identified the gut microbiome of urban and rural Russian populations. ${ }^{25}$ Based on the findings of these studies, the diet, genetic differences, mode of delivery, lifestyle, medical treatment, and immune status of the host have been suggested as factors affecting the community structure of the gut microbiome..$^{21-25}$ In contrast, the stable community structure of gut microbiota was detected in different human populations in a very recent study. ${ }^{26}$ Furthermore, the presence of a certain degree of functional redundancy is suggested with similar protein or metabolic profiles of gut microbiome, irrespective of their compositional variations. ${ }^{27}$

Compositional changes of entire microbial communities in disease conditions compared to healthy status have been explained by the concept of microbial dysbiosis, a fundamental concept in microbial ecology. ${ }^{28,29}$ Furthermore, case control studies comparing the fecal microbiota of healthy individuals with those of patients have found microbial dysbiosis associated with the etiology or progression of a range of GI diseases such as Crohn's disease, ${ }^{30}$ irritable bowel syndrome (IBS), ${ }^{31}$ colon cancer, ${ }^{32}$ and antibiotic-associated diarrhea (ADD). ${ }^{33}$ More recent evidence has suggested the involvement of the gut microbiome in conditions like obesity, $^{34,35}$ diabetes ${ }^{35,36}$ as well as brain development and behavior. ${ }^{37}$

\section{Role of the Gut Microbiome in Health}

The microbiota inhabiting the gut regulate important physiological functions in the host such as strengthening the gut integrity, ${ }^{38}$ digestion and extraction of energy from food, ${ }^{39}$ breakdown of toxins and xenobiotics, production of vitamins and essential immuno acids, protection against pathogens of the host, and modification of neurological development and behavior. ${ }^{5,10}$ Furthermore, cross-talk between the gut commensal bacteria and the mucosal immune system are essential for shaping the host's immunity. ${ }^{40}$ Interestingly, the gut microbiota plays a crucial role in human metabolism by supplying enzymes that are not encoded by the human genome, for example the breakdown of polysaccharides, polyphenols, and synthesis of vitamins. ${ }^{41}$ In vitro studies using human fecal incubations or more complex continuous culture methods, comparative studies in germ free and conventional microbiota, as well as in vivo studies of animal models have provided the laboratory evidence for the role of the gut microbiome in the metabolism of dietary components and its impact on health. ${ }^{41}$

Colonic bacteria are capable of expressing carbohydrateactive enzymes, which ferment complex carbohydrates, and generating metabolites for example short chain fatty acids (SCFAs). ${ }^{35}$ Propionate, butyrate, and acetate are predominant among them, and they are found in a proportion of 1:1:3 under healthy conditions in the GI tract. ${ }^{42}$ These SCFAs are promptly absorbed by epithelial cells in the GI tract. These SCFAs are 
implicated in the regulation of cellular processes such as gene expression, chemotaxis, differentiation, proliferation, and apoptosis in the host. ${ }^{42}$ Most gut anaerobes produce acetate. In contrast, propionate and butyrate are produced by a different subset of gut bacteriome via distinct molecular pathways. ${ }^{43}$ The fermentation of starch by specialist Actinobacteria and Firmicutes is suggested to contribute extensively to butyrate production in the colon directly and by cross-feeding. ${ }^{44}$ Of them, the main propionate producer Akkermansia muciniphila is specialized in mucin degradation. ${ }^{45}$ The functions of SCFAs in human metabolism have been reviewed. ${ }^{46,47}$ Butyrate has shown anti-inflammatory and anti-cancer activities. ${ }^{47}$ This is an important energy source for colonocytes and can attenuate bacterial translocation by enhancing gut barrier functions. ${ }^{42}$ SCFAs are responsible for regulating hepatic lipid and glucose homeostasis by way of harmonizing mechanisms. Furthermore, SCFAs contribute to the regulation of the immune system and inflammatory responses. ${ }^{46}$ They stimulate the production of IL-18, an interleukin involved in maintaining and repairing epithelial integrity. ${ }^{48}$ Butyrate and propionate can act as histone deacetylase inhibitors to epigenetically regulate gene expression. ${ }^{46,47}$ Other microbial metabolites have also emerged as important compounds as they have effects on intestinal barrier functions, epithelium proliferation, and the immune system. ${ }^{49}$

The gut microbiome plays a key role in the de novo synthesis of essential vitamins in the human gut. ${ }^{50}$ Vitamin B12 is mainly synthesized by neither Lactic acid bacteria, neither produce by animals, plants nor fungi. ${ }^{50,51}$ Folate is a vitamin involved in DNA synthesis and repair. Bifidobacteria are the number one producer of folate in the human gut. ${ }^{52}$ Other vitamins synthesized by the gut microbiome are vitamin $\mathrm{K}$, riboflavin, biotin, nicotinic acid, panthotenic acid, pyridoxine, and thiamin..$^{53}$ Moreover, colonic bacteria are responsible for metabolizing bile acids. ${ }^{54}$ The homeostasis of all these metabolic processes helps the host be healthy. Thus, alterations in the co-metabolism of bile acids, branched fatty acids, choline, purine, vitamins, and phenolic compounds have been associated with the development of metabolic diseases such as obesity and type 2 diabetes. ${ }^{55}$

The influence of the GI microbiome in the development of both intestinal mucosal and systemic immune systems has been demonstrated by experiments on germ-free animals. In these experiments, the immune deficiency was completely reversed by the treatment of germ-free (GF) mice with polysaccharide A from the capsule of Bacteroides fragilis. ${ }^{56}$ Segmented filamentous bacteria (SBF) is a class of anaerobic and Clostridia-related, spore-forming commensals harbored in the mammalian GI tract that interacts dynamically with the immune system. ${ }^{57} \mathrm{~A}$. muciniphila has been implicated with protection against several inflammatory diseases, ${ }^{58,59}$ suggestive of anti-inflammatory properties, but the underlying mechanisms are yet to be identified ${ }^{60}$ Hence, individuals with Crohn's disease demonstrate mucosal dysbiosis characterized by reduced diversity of core microbiota and a lower abundance of Faecalibacterium prausnitzii. ${ }^{60}$ Recently in an animal model, the anti-inflammatory protein from $F$. prausnitzii was shown to inhibit the NF- $\kappa \mathrm{B}$ pathway in intestinal epithelial cells and prevent colitis. ${ }^{61}$

There is substantial laboratory evidence supporting the role of the gut microbiome in influencing epithelial homeostasis. ${ }^{38}$ Impaired epithelial cell turnover in germ-free mice has been reversible upon colonization of microbiota. ${ }^{62}$ Bacteria such as A. muciniphila ${ }^{63}$ and Lactobacillus plantarum ${ }^{64}$ have been associated with promoting epithelial integrity. In addition, it has been proposed that bacteria modulate mucus properties in experiments using germ-free mouse models. These mice have extremely thin adherent colonic mucus layers. Nevertheless, when exposed to bacterial peptidoglycan or lipopolysaccharides, the thickness of the adherent mucus layer is restored to levels of conventionally reared mice. ${ }^{65}$ These functions mediate a competitive advantage to other commensals in colonization. ${ }^{66}$ Moreover, commensal microbiota in the human gut are capable of controlling the colonization of pathogens by competing for attachment sites or nutrient sources and by producing antimicrobial substances such as bacteriocins ${ }^{8,67}$ in competitive exclusion. ${ }^{65}$

\section{Adverse Effects of Antibiotics on the Healthy Gut Microbiome} Brief Introduction to Antibiotics and Antibiotic Resistance Antibiotics are a class of antimicrobials which act against bacteria. Since the Second World War, antibiotics have become the cornerstone in medicine. It has been estimated that the usage of antibiotics has extended man's life expectancy by an average of 20 years. ${ }^{68}$ Furthermore, antimicrobials have been widely used in the poultry, animal husbandry, and shrimp industries for therapeutic purposes, disease prevention, and growth promotion. ${ }^{6}$

Antimicrobials have existed in nature for hundreds of millions of years. Bacteriocins are one example of such naturally occurring antibiotics. They are active against other bacteria to which the producer is immune. ${ }^{70}$ In natural environments these antimicrobial substances may contribute to competitive exclusion of other bacteria which compete for scarce resources. Hence, selective benefit is provided for the producing strain. ${ }^{71,72}$ Resistance to antibiotics may occur in a population of susceptible bacteria by the accumulation of mutations (point mutations in DNA gyrase presenting resistance to quinolones) or by the acquisition of a resistance gene. ${ }^{73}$ Phenotypic resistance is the outcome of the presence of resistant genes in bacteria, and it acts through a variety of mechanisms such as enzymatic inactivation of the antibiotic, modification of the target of the antibiotic, and prevention of the accumulation of lethal intra-cellular concentrations of the antibiotic through efflux pumps. ${ }^{74}$ The history of the presence of resistance and the emergence of the serine $\beta$ lactamases dates back 2 billion years. ${ }^{75}$

Disturbance to the Ecology of the Healthy Gut Microbiome Disturbance to ecology is defined as an event or process (physical or biological) that makes sudden structural changes in community composition. ${ }^{76}$ The adverse effects of the use of massive quantities and concentrations of antibiotics can cause dysbiosis in human indigenous microbiota in 
individual subjects. Antibiotic-associated microbial dysbiosis is an emerging concept; thus, relatively few studies have addressed this matter using NGS approaches with frequent sampling in cohort studies. ${ }^{76}$ It has been revealed that broad spectrum antibiotics can cause microbial dysbiosis, affecting the abundance of $30 \%$ of bacteria in the gut community, resulting in significant drops in taxonomic richness, diversity, and evenness. ${ }^{28,77}$ Depletion of bacterial diversity and loss of potential competitors, lower expression of antibacterials, and IgG as well as decreased neutrophil-mediated killing may enhance susceptibility by exogenous pathogens or by opportunistic members of the microbiota. ${ }^{78,79}$ Antibiotics with strong anti-anaerobic activity such as clindamycin cause a decline in Bacteroides diversity after 7 days of use, which could persist for at least 2 years in healthy volunteers. ${ }^{80,81}$

Use of an antibiotic regimen with clarithromycin and metronidazole affected the fecal microbiota of healthy individuals for 4 years in another study. ${ }^{82}$ Effects of a 5-day (pulse) exposure to ciprofloxacin on healthy adult volunteers with a sampling scheme that included monthly, then weekly, then daily fecal specimens for two months prior to exposure, and then the reverse for 6 months after exposure, were assessed by pyrosequencing the $16 \mathrm{~S}$ rRNA hypervariable region in two studies. ${ }^{28,77}$ A 5-day exposure to ciprofloxacin can lead to a sudden decline in diversity by the end of the exposure period and a marked change in the relative abundance of approximately one third to one half of all taxa. The taxa most affected consisted of members of Ruminococcaceae and Lachnospiraceae within the phylum Firmicutes and Bacteroidetes, respectively. ${ }^{28}$ Nevertheless, by 2 weeks following exposure, they returned to pre-exposure abundance levels. In general, the second exposure caused a similar perturbation and less complete recovery to the pre-exposure level. Each subject showed individualized responses. ${ }^{28}$ These findings suggest that the repeated use of antibiotics can slow down the recovery of the gut microbiome to pre-exposure level. ${ }^{28}$

Studies have shown that beyond altering the composition of taxa, antibiotics can affect the gene expression, protein activity, and overall metabolism of the gut microbiome. These changes can occur at a much faster pace than replacing the microbiome to the pre-exposure state. ${ }^{83}$ Thus, antibiotics have an influence on gut microbiome that pave the way to changes in the nutritional landscape of the gut, directing the expansion of pathogenic bacteria. ${ }^{84}$ Commensal gut microbiota liberates increased levels of sialic acid during xenobiotic metabolism after consumption of antibiotics. This situation will favor opportunistic pathogens such as S. typhimurium and $C$. difficile who are versatile in utilizing fucose and salic acid compared to the gut commensals. Thus, post-antibiotic treatment facilitates their expansion within the gut. ${ }^{85}$ The induced changes can lead the functionality of microbes near to states similar to those monitored under disease states. The gut microbiome of individuals treated with $\beta$-lactams demonstrated enzyme activities for carbohydrate degradation that resulted in an unbalanced sugar metabolism similar to that observed in obese individuals. ${ }^{86}$ There was an increased proportion of gut microbial cells with damaged membranes in ex-vivo incubation of fecal samples with different antibiotics. The active populations of the microbiota changed and genes involved in antibiotic resistance, stress response, and phage induction triggered the expression of fecal microbiota in the same experiment. ${ }^{86}$

\section{Increased Susceptibility to Infections}

Increased susceptibility to infections by a newly acquired pathogen or opportunistic ones (with sudden overgrowth and pathogenic behavior) of the gut microbiome could occur because of alterations in the gut microbiome due to the use of antibiotics. In particular, AAD due to nosocomial pathogens such as Clostridium difficile can cause intractable long-term recurrent infections and more dangerous pseudomembranous colitis ${ }^{87}$. Furthermore, enteric bacteria, namely Escherichia coli, Klebsiella pneumoniae, Enerococcus faecalis, and Enerococcus faecum, have emerged as multidrugresistant nosocomial pathogens of major importance due to the irrational use of antibiotics. ${ }^{88,89}$ Opportunistic pathogens from the gut become pathogenic by translocating across the intestinal barrier, and fecal contamination of skin and other body sites might cause nosocomial infections by placement of a catheter or an intravenous line. ${ }^{88,89}$

\section{Accumulation of Resistance}

The human gut microbiota has been established as a significant reservoir for ARGs. ${ }^{90,91}$ The gut microbiota provides opportunities for the horizontal transfer of genetic material including antibiotic resistance genes. Shotgun sequencing techniques have allowed the extensive study of antibiotic resistance genes that are harbored by the gut microbiota ("the gut resistome"). Genes conferring resistance to antibiotics are ubiquitously present among the gut microbiota of humans, and most resistance genes are harbored by strictly anaerobic gut commensals. ${ }^{9}$ Several metagenomic studies have been done to probe the resistome in the gut of healthy ${ }^{92,93,94,95}$ and hospitalized individuals. ${ }^{96,97}$ Resistance genes for 50 of 68 classes of antibiotics in 252 fecal metagenomes, at an average of 21 antibiotic resistance genes per sample, were obtained in a study conducted by Forslund et al. ${ }^{92}$ Samples were obtained from individuals in Spain, Italy, Denmark, France, Malawi, the United States, and Japan. This largest population study on gut resistome to date showed that the abundance of resistance genes (ARGs) was greatest for antibiotics that have been sold for a long time and for those approved for animal use, such as tetracycline and bacitracin. Individuals from countries such as Denmark (with restrained policies of antibiotics use in humans and animals) harbored lower levels of resistance genes in the gut microbiome. European samples demonstrated enhanced resistance to vancomycin whereas the sample from the United States. In the United States, a vancomycin analog is used to treat animals. These observations endorse the notion that a higher exposure to antibiotics elevates the likelihood of resistome formation in the gut microbiome. In another study conducted by $\mathrm{Hu}$ et $\mathrm{al},{ }^{93}$ a total of 1093 antibiotic resistance genes in 162 individuals from China and Denmark were 
obtained. Genes resistant to the antibiotic tetracycline (tet 32, tet 40 , tet $\mathrm{O}$, tet $\mathrm{Q}$, and tet $\mathrm{W}$ ) were present in the microbiota of all individuals. These are encountered as the most abundant family of resistance genes in both studies described previously. ${ }^{92,93}$ Other resistance genes that were ubiquitously present conferred resistance to aminoglycosides (ant (6')-IA), bacitracin (bac $A$ ), and the glycopeptides like vancomycin (van $R A$ and van $R G)^{93}$. Furthermore, the geographic specificity of antibiotic resistance genes was demonstrated by single nucleotide polymorphisms (SNPs). ${ }^{98}$ Regional variations in the sequence and abundance of resistance genes might be associated with antibiotic type and exposure of the microbiota in China and European countries. Further studies with large sample sizes and representing other countries, especially South Asian developing countries which carry a huge burden of antibiotic resistance and were not included in previous studies, are much warranted to confirm these findings.

The transfer of ARGs among gut microbiome Bacteroides as well as the horizontal transfer from Bacteroides and gram positive bacteria have been documented. ${ }^{99}$ Experimental evidence based on rats and mice has confirmed the transfer of ARG-carrying transposons between gut bacteria. ${ }^{100,} 101$ Low concentrations of antibiotics (100-1000 fold) stimulates the transfer of conjugative transposons $s^{102}$ between gut bacteriomes. ARGs harbored in gut microbiota could be transferred to incoming pathogens. Many of the resistant genes identified from human gut isolates are identical at the nucleotide level to resistance genes from human pathogen ${ }^{95}$ and the gut microbiome acts as a reservoir for ARGs. ${ }^{12}$

Mechanisms such as conjugation, transformation, and transduction can cause the transfer of antibiotic resistance genes and the spread of ARGs in the gut microbiome. ${ }^{103}$ In transformation, naked DNA which might have antibiotic resistance genes is taken up by a bacterium. ${ }^{103}$ During conjugative transfer, resistance genes could spread after the formation of a mating bridge between two cells. In this process, plasmids or conjugative transposons will move from a donor cell to a recipient. In transduction, resistance genes that are encoded by bacteriophage can transfer from one cell to another and be integrated into the chromosome of the recipient cell in the process termed lysogeny. ${ }^{103}$ Transformation does not contribute significantly to horizontal gene transfer in the mammalian intestinal tract. ${ }^{104}$ However, both conjugation and transduction are responsible for the horizontal transfer of ARGs. Thus, the human gut is an important site for the accumulation of ARGs and for their expansion across species. ${ }^{12}$

In healthy individuals, nonpathogenic commensals dominate in the gut microbiome, and the horizontal transfer of genetic material is a common event in the gut. ${ }^{9}$ Usually, ARGs transfer from commensals to gut-inhabiting pathogens might be a rare event. Nevertheless, this possibility cannot be ignored when considering the emergence of multidrugresistant strains. An example is the vancomycin resistance determinants that are shared by anaerobic gut commensals and the nosocomial pathogen Enterococcus faecium. ${ }^{9}$ When antibiotics are essential as a lifesaving treatment, modality benefits outweigh the risks of the adverse effects of antibiotics. Nevertheless, the adverse effects of irrational use of antibiotics could be prevented by primordial preventive measures where cons outweigh the pros.

\section{Conclusions}

There is an emerging interest in the adverse effects of antibiotics on the healthy gut microbiome as evident from the increasing number of recent publications related to the topic of this mini-review. Nevertheless, published studies disagree on the potential mechanisms by which gene expression, protein activity, and overall metabolic changes occur in the gut microbiome, which may influence the normal metabolic and immunological functions of the host as well as how long the gut microbiome takes to return to the original level. This disagreement could be partially attributed to major methodological variations in metagenomic and metatranscriptomic studies with respect to sampling techniques, DNA extraction techniques, and microbial profiling technologies, the selection of primers and hypervariable regions for amplifications and bioinformatics. Thus, future work is much warranted, in addition to methodological standardization, including complete metagenomics, metatranscriptomic, mechanistic studies using animal models and in vitro studies to understand all adverse effects of antibiotics on normal gut microbiome. Banning the irrational use of antibiotics, public health interventions to promote the rational use of antibiotics, and extensive investigations into the environmental and sociocultural drivers of antibiotic resistomes may help reduce the occurrence of multidrug-resistant strains in the future.

Moreover, there is a need for corroborative studies conducted in developing countries which carry a high burden of antibiotic resistance compounded by many knowledge gaps. This emerging research arena has a promising future as many scientists are keen on developing interventions in randomized clinical trials with the use of pre-biotics and probiotics to return the altered, dysbiotic gut microbiome to its original, healthy state, subsequent to exposure to antibiotics in the modern era of personalized medicine.

\section{Authors' Contributions}

All authors contributed equally to this study.

\section{Conflict of Interest Disclosures}

The authors declare that they have no conflicts of interest.

\section{References}

1. Turnbaugh PJ, Ley RE, Hamady M, Fraser-Liggett CM, Knight $\mathrm{R}$, Gordon Jl. The human microbiome project. Nature. 2007;449(7164):804-810. doi:10.1038/nature06244.

2. Parahitiyawa NB, Scully C, Leung WK, Yam WC, Jin LJ, Samaranayake LP. Exploring the oral bacterial flora: current status and future directions. Oral Dis. 2010;16(2):136-145. doi:10.1111/ j.1601-0825.2009.01607.x.

3. Ling Z, Kong J, Jia P, et al. Analysis of oral microbiota in children with dental caries by PCR-DGGE and barcoded pyrosequencing. Microb Ecol. 2010;60(3):677-690. doi:10.1007/s00248-010- 
9712-8.

4. Kilian M, Chapple IL, Hannig M, et al. The oral microbiome - an update for oral healthcare professionals. $\mathrm{Br}$ Dent J. 2016;221(10):657-666. doi:10.1038/sj.bdj.2016.865.

5. Sonnenburg JL, Fischbach MA. Community health care: therapeutic opportunities in the human microbiome. Sci Transl Med. 2011;3(78):78ps12. doi:10.1126/scitranslmed.3001626.

6. Structure, function and diversity of the healthy human microbiome. Nature.2012;486(7402):207-214.doi:10.1038/nature11234.

7. Huse SM, Ye Y, Zhou Y, Fodor AA. A core human microbiome as viewed through $16 \mathrm{~S}$ rRNA sequence clusters. PLoS One. 2012;7(6):e34242. doi:10.1371/journal.pone.0034242.

8. Thursby $\mathrm{E}$, Juge N. Introduction to the human gut microbiota. Biochem J. 2017;474(11):1823-1836. doi:10.1042/bcj20160510.

9. van Schaik W. The human gut resistome. Philos Trans R Soc Lond B Biol Sci. 2015;370(1670):20140087. doi:10.1098/rstb.2014.0087.

10. Gensollen T, lyer SS, Kasper DL, Blumberg RS. How colonization by microbiota in early life shapes the immune system. Science. 2016;352(6285):539-544. doi:10.1126/science.aad9378.

11. Francino MP. Early development of the gut microbiota and immune health. Pathogens. 2014;3(3):769-790. doi:10.3390/ pathogens3030769.

12. Francino MP. Antibiotics and the human gut microbiome: dysbioses and accumulation of resistances. Front Microbiol. 2015;6:1543. doi:10.3389/fmicb.2015.01543.

13. Kinch MS, Patridge E, Plummer M, Hoyer D. An analysis of FDA-approved drugs for infectious disease: antibacterial agents. Drug Discov Today. 2014;19(9):1283-1287. doi:10.1016/j. drudis.2014.07.005.

14. O'Neill J. Antimicrobial resistance: tackling a crisis for the health and wealth of nations. The Review on Antimicrobial Resistance. 2014 . https://amr-review.org/sites/default/files/AMR\%20 Review\%20Paper\%20-\%20Tackling\%20a\%20crisis\%20for\%20 the $\% 20$ health $\% 20$ and $\% 20$ wealth $\% 20$ of\%20nations_1.pdf.

15. Franzosa EA, Hsu T, Sirota-Madi A, et al. Sequencing and beyond: integrating molecular 'omics' for microbial community profiling. Nat Rev Microbiol. 2015;13(6):360-372. doi:10.1038/ nrmicro3451.

16. Moore WE, Holdeman LV. Human fecal flora: the normal flora of 20 Japanese-Hawaiians. Appl Microbiol. 1974;27(5):961-979.

17. Suau A, Bonnet $R$, Sutren $M$, et al. Direct analysis of genes encoding 16S rRNA from complex communities reveals many novel molecular species within the human gut. Appl Environ Microbiol. 1999;65(11):4799-4807.

18. Poretsky R, Rodriguez RL, Luo C, Tsementzi D, Konstantinidis KT. Strengths and limitations of $16 \mathrm{~S}$ rRNA gene amplicon sequencing in revealing temporal microbial community dynamics. PLoS One. 2014;9(4):e93827. doi:10.1371/journal.pone.0093827.

19. Mizrahi-Man O, Davenport ER, Gilad Y. Taxonomic classification of bacterial $16 \mathrm{~S}$ rRNA genes using short sequencing reads: evaluation of effective study designs. PLoS One. 2013;8(1):e53608. doi:10.1371/journal.pone.0053608.

20. Hugon P, Dufour JC, Colson P, Fournier PE, Sallah K, Raoult D. A comprehensive repertoire of prokaryotic species identified in human beings. Lancet Infect Dis. 2015;15(10):1211-1219. doi:10.1016/s1473-3099(15)00293-5.

21. Li J, Jia H, Cai X, et al. An integrated catalog of reference genes in the human gut microbiome. Nat Biotechnol. 2014;32(8):834-841. doi:10.1038/nbt.2942.

22. Rajilic-Stojanovic M, de Vos WM. The first 1000 cultured species of the human gastrointestinal microbiota. FEMS Microbiol Rev. 2014;38(5):996-1047. doi:10.1111/1574-6976.12075.

23. Zhang Q, Raoof $M$, Chen $Y$, et al. Circulating mitochondrial DAMPs cause inflammatory responses to injury. Nature. 2010;464(7285):104-107.

doi:10.1038/nature08780.

24. Tyakht AV, Kostryukova ES, Popenko AS, et al. Human gut microbiota community structures in urban and rural populations in Russia. Nat Commun. 2013;4:2469. doi:10.1038/ncomms3469.
25. Jackson MA, Bonder MJ, Kuncheva Z, et al. Detection of stable community structures within gut microbiota co-occurrence networks from different human populations. PeerJ. 2018;6:e4303. doi:10.7717/peerj.4303.

26. Moya A, Ferrer M. Functional Redundancy-Induced Stability of Gut Microbiota Subjected to Disturbance. Trends Microbiol. 2016;24(5):402-413. doi:10.1016/j.tim.2016.02.002.

27. Dethlefsen L, Relman DA. Incomplete recovery and individualized responses of the human distal gut microbiota to repeated antibiotic perturbation. Proc Natl Acad Sci U S A. 2011;108 Suppl 1:45544561. doi:10.1073/pnas.1000087107.

28. Perera M, Al-Hebshi NN, Perera I, et al. Inflammatory bacteriome and oral squamous cell carcinoma. J Dent Res. 2018;97(6):725732. doi:10.1177/0022034518767118.

29. Manichanh C, Rigottier-Gois L, Bonnaud E, et al. Reduced diversity of faecal microbiota in Crohn's disease revealed by a metagenomic approach. Gut. 2006;55(2):205-211. doi:10.1136/ gut.2005.073817.

30. Kassinen A, Krogius-Kurikka L, Makivuokko H, et al. The fecal microbiota of irritable bowel syndrome patients differs significantly from that of healthy subjects. Gastroenterology. 2007;133(1):2433. doi:10.1053/j.gastro.2007.04.005.

31. Scanlan PD, Shanahan F, Clune Y, et al. Culture-independent analysis of the gut microbiota in colorectal cancer and polyposis. Environ Microbiol. 2008;10(3):789-798. doi:10.1111/j.14622920.2007.01503.x.

32. Young VB, Schmidt TM. Antibiotic-associated diarrhea accompanied by large-scale alterations in the composition of the fecal microbiota. J Clin Microbiol. 2004;42(3):1203-1206. doi:10.1128/JCM.42.3.1203-1206.2004.

33. Greenblum S, Turnbaugh PJ, Borenstein E. Metagenomic systems biology of the human gut microbiome reveals topological shifts associated with obesity and inflammatory bowel disease. Proc Natl Acad Sci U S A. 2012;109(2):594-599. doi:10.1073/ pnas. 1116053109.

34. Musso G, Gambino R, Cassader M. Obesity, diabetes, and gut microbiota: the hygiene hypothesis expanded? Diabetes Care. 2010;33(10):2277-2284. doi:10.2337/dc10-0556.

35. Marchesi JR, Adams DH, Fava F, et al. The gut microbiota and host health: a new clinical frontier. Gut. 2016;65(2):330-339. doi:10.1136/gutjnl-2015-309990.

36. Diaz Heijtz R, Wang S, Anuar F, et al. Normal gut microbiota modulates brain development and behavior. Proc Natl Acad Sci U S A. 2011;108(7):3047-3052. doi:10.1073/pnas.1010529108.

37. Natividad JM, Verdu EF. Modulation of intestinal barrier by intestinal microbiota: pathological and therapeutic implications. Pharmacol Res.2013;69(1):42-51.doi:10.1016/j.phrs.2012.10.007.

38. den Besten G, van Eunen K, Groen AK, Venema K, Reijngoud DJ, Bakker BM. The role of short-chain fatty acids in the interplay between diet, gut microbiota, and host energy metabolism. J Lipid Res. 2013;54(9):2325-2340. doi:10.1194/jlr.R036012.

39. Tomkovich S, Jobin C. Microbiota and host immune responses: a love-hate relationship. Immunology. 2016;147(1):1-10. doi:10.1111/imm.12538.

40. Rowland I, Gibson G, Heinken A, et al. Gut microbiota functions: metabolism of nutrients and other food components. Eur J Nutr. 2018;57(1):1-24. doi:10.1007/s00394-017-1445-8.

41. Louis P, Hold GL, Flint HJ. The gut microbiota, bacterial metabolites and colorectal cancer. Nat Rev Microbiol. 2014;12(10):661-672. doi:10.1038/nrmicro3344.

42. Louis $\mathrm{P}$, Scott KP, Duncan SH, Flint HJ. Understanding the effects of diet on bacterial metabolism in the large intestine. J Appl Microbiol. 2007;102(5):1197-1208. doi:10.1111/j.13652672.2007.03322.x.

43. Louis P, Flint HJ. Formation of propionate and butyrate by the human colonic microbiota. Environ Microbiol. 2017;19(1):29-41. doi:10.1111/1462-2920.13589.

44. Derrien M, Vaughan EE, Plugge CM, de Vos WM. Akkermansia 
muciniphila gen. nov., sp. nov., a human intestinal mucindegrading bacterium. Int J Syst Evol Microbiol. 2004;54(Pt 5):1469-1476. doi:10.1099/ijs.0.02873-0.

45. Morrison DJ, Preston T. Formation of short chain fatty acids by the gut microbiota and their impact on human metabolism. Gut Microbes. 2016;7(3):189-200. doi:10.1080/19490976.2015.113 4082

46. Lin L, Zhang J. Role of intestinal microbiota and metabolites on gut homeostasis and human diseases. BMC Immunol. 2017;18(1):2. doi:10.1186/s12865-016-0187-3.

47. Correa-Oliveira R, Fachi JL, Vieira A, Sato FT, Vinolo MA. Regulation of immune cell function by short-chain fatty acids. Clin Transl Immunology. 2016;5(4):e73. doi:10.1038/cti.2016.17.

48. Nagai M, Obata Y, Takahashi D, Hase K. Fine-tuning of the mucosal barrier and metabolic systems using the diet-microbial metabolite axis. Int Immunopharmacol. 2016;37:79-86. doi:10.1016/j. intimp.2016.04.001

49. LeBlanc JG, Milani C, de Giori GS, Sesma F, van Sinderen D, Ventura M. Bacteria as vitamin suppliers to their host: a gut microbiota perspective. Curr Opin Biotechnol. 2013;24(2):160168. doi:10.1016/j.copbio.2012.08.005.

50. Martens JH, Barg H, Warren MJ, Jahn D. Microbial production of vitamin B12. Appl Microbiol Biotechnol. 2002;58(3):275-285. doi:10.1007/s00253-001-0902-7.

51. Pompei A, Cordisco L, Amaretti A, Zanoni S, Matteuzzi D, Rossi M. Folate production by bifidobacteria as a potential probiotic property. Appl Environ Microbiol. 2007;73(1):179-185. doi:10.1128/aem.01763-06.

52. Hill MJ. Intestinal flora and endogenous vitamin synthesis. Eur J Cancer Prev. 1997;6 Suppl 1:S43-45. doi:10.1097/00008469199703001-00009.

53. Staley C, Weingarden AR, Khoruts A, Sadowsky MJ. Interaction of gut microbiota with bile acid metabolism and its influence on disease states. Appl Microbiol Biotechnol. 2017;101(1):47-64. doi:10.1007/s00253-016-8006-6.

54. Palau-Rodriguez M, Tulipani S, Isabel Queipo-Ortuno M, UrpiSarda M, Tinahones FJ, Andres-Lacueva C. Metabolomic insights into the intricate gut microbial-host interaction in the development of obesity and type 2 diabetes. Front Microbiol. 2015;6:1151. doi:10.3389/fmicb.2015.01151.

55. Mazmanian SK, Liu CH, Tzianabos AO, Kasper DL. An immunomodulatory molecule of symbiotic bacteria directs maturation of the host immune system. Cell. 2005;122(1):107118. doi:10.1016/j.cell.2005.05.007.

56. Schnupf P, Gaboriau-Routhiau V, Gros M, et al. Growth and host interaction of mouse segmented filamentous bacteria in vitro. Nature. 2015;520(7545):99-103. doi:10.1038/nature14027.

57. Everard A, Belzer C, Geurts L, et al. Cross-talk between Akkermansia muciniphila and intestinal epithelium controls dietinduced obesity. Proc Natl Acad Sci U S A. 2013;110(22):90669071. doi:10.1073/pnas.1219451110.

58. Wang L, Christophersen CT, Sorich MJ, Gerber JP, Angley MT, Conlon MA. Low relative abundances of the mucolytic bacterium Akkermansia muciniphila and Bifidobacterium spp. in feces of children with autism. Appl Environ Microbiol. 2011;77(18):67186721. doi:10.1128/aem.05212-11.

59. Sokol H, Seksik P, Furet JP, et al. Low counts of Faecalibacterium prausnitzii in colitis microbiota. Inflamm Bowel Dis. 2009;15(8):1183-1189. doi:10.1002/ibd.20903.

60. Quevrain E, Maubert MA, Michon C, et al. Identification of an anti-inflammatory protein from Faecalibacterium prausnitzii, a commensal bacterium deficient in Crohn's disease. Gut. 2016;65(3):415-425. doi:10.1136/gutjnl-2014-307649.

61. Smith K, McCoy KD, Macpherson AJ. Use of axenic animals in studying the adaptation of mammals to their commensal intestinal microbiota. Semin Immunol. 2007;19(2):59-69. doi:10.1016/j. smim.2006.10.002.

62. Reunanen J, Kainulainen V, Huuskonen L, et al. Akkermansia muciniphila Adheres to Enterocytes and Strengthens the
Integrity of the Epithelial Cell Layer. Appl Environ Microbiol. 2015;81(11):3655-3662. doi:10.1128/aem.04050-14.

63. Chen HQ, Yang J, Zhang M, et al. Lactobacillus plantarum ameliorates colonic epithelial barrier dysfunction by modulating the apical junctional complex and PepT1 in IL-10 knockout mice. Am J Physiol Gastrointest Liver Physiol. 2010;299(6):G1287-1297. doi:10.1152/ajpgi.00196.2010.

64. Petersson J, Schreiber O, Hansson GC, et al. Importance and regulation of the colonic mucus barrier in a mouse model of colitis. Am J Physiol Gastrointest Liver Physiol. 2011;300(2):G327333. doi:10.1152/ajpgi.00422.2010.

65. Freitas M, Cayuela C, Antoine JM, Piller F, Sapin C, Trugnan G. A heat labile soluble factor from Bacteroides thetaiotaomicron VPI5482 specifically increases the galactosylation pattern of HT29MTX cells. Cell Microbiol. 2001;3(5):289-300. doi:10.1046/ j.1462-5822.2001.00113.x.

66. Baumler AJ, Sperandio V. Interactions between the microbiota and pathogenic bacteria in the gut. Nature. 2016;535(7610):85-93 doi:10.1038/nature18849.

67. Mandal S, Mishra S, Mandal PK. Microbiome and Antimicrobial Resistance: A Mini Review. J Biotechnol Bioeng. 2017;1(1):14-16.

68. Hawkey PM. The growing burden of antimicrobial resistance. J Antimicrob Chemother. 2008;62 Suppl 1:i1-9. doi:10.1093/jac/ $\mathrm{dkn} 241$.

69. Hegarty JW, Guinane CM, Ross RP, Hill C, Cotter PD. Bacteriocin production: a relatively unharnessed probiotic trait? F1000Res. 2016;5:2587. doi:10.12688/f1000research.9615.1.

70. Aminov RI. The role of antibiotics and antibiotic resistance in nature. Environ Microbiol. 2009;11(12):2970-2988. doi:10.1111/ j.1462-2920.2009.01972.x.

71. Martinez JL. Antibiotics and antibiotic resistance genes in natural environments. Science. 2008;321(5887):365-367. doi:10.1126/ science. 1159483.

72. Allen HK, Donato J, Wang HH, Cloud-Hansen KA, Davies J, Handelsman J. Call of the wild: antibiotic resistance genes in natural environments. Nat Rev Microbiol. 2010;8(4):251-259. doi:10.1038/nrmicro2312.

73. Martinez JL. General principles of antibiotic resistance in bacteria. Drug Discov Today Technol. 2014;11:33-39. doi:10.1016/j. ddtec.2014.02.001.

74. Hall BG, Barlow M. Evolution of the serine beta-lactamases: past, present and future. Drug Resist Updat. 2004;7(2):111-123. doi:10.1016/j.drup.2004.02.003.

75. Relman DA. The human microbiome: ecosystem resilience and health. Nutr Rev. 2012;70 Suppl 1:S2-9. doi:10.1111/j.17534887.2012.00489.x.

76. Dethlefsen L, Huse S, Sogin ML, Relman DA. The pervasive effects of an antibiotic on the human gut microbiota, as revealed by deep 16S rRNA sequencing. PLoS Biol. 2008;6(11):e280. doi:10.1371/ journal.pbio.0060280.

77. Ubeda C, TaurY, Jenq RR, et al. Vancomycin-resistant Enterococcus domination of intestinal microbiota is enabled by antibiotic treatment in mice and precedes bloodstream invasion in humans. J Clin Invest. 2010;120(12):4332-4341. doi:10.1172/jci43918.

78. Madan JC, Salari RC, Saxena D, et al. Gut microbial colonisation in premature neonates predicts neonatal sepsis. Arch Dis Child Fetal Neonatal Ed. 2012;97(6):F456-462. doi:10.1136/ fetalneonatal-2011-301373.

79. Jernberg C, Lofmark S, Edlund C, Jansson JK. Long-term ecological impacts of antibiotic administration on the human intestinal microbiota. Isme j. 2007;1(1):56-66. doi:10.1038/ismej.2007.3.

80. Jernberg C, Lofmark S, Edlund C, Jansson JK. Long-term impacts of antibiotic exposure on the human intestinal microbiota. Microbiology. 2010;156(Pt 11):3216-3223. doi:10.1099/ mic.0.040618-0.

81. Jakobsson $\mathrm{HE}$, Jernberg $\mathrm{C}$, Andersson $\mathrm{AF}$, Sjolund-Karlsson $\mathrm{M}$, Jansson JK, Engstrand L. Short-term antibiotic treatment has differing long-term impacts on the human throat and gut microbiome. PLoS One. 2010;5(3):e9836. doi:10.1371/journal.pone.0009836. 
82. Perez-Cobas AE, Gosalbes MJ, Friedrichs A, et al. Gut microbiota disturbance during antibiotic therapy: a multi-omic approach. Gut. 2013;62(11):1591-1601. doi:10.1136/gutjnl-2012-303184.

83. Ferreyra JA, Wu KJ, Hryckowian AJ, Bouley DM, Weimer BC, Sonnenburg JL. Gut microbiota-produced succinate promotes $C$. difficile infection after antibiotic treatment or motility disturbance. Cell Host Microbe. 2014;16(6):770-777. doi:10.1016/j. chom.2014.11.003.

84. Ng KM, Ferreyra JA, Higginbottom SK, et al. Microbiota-liberated host sugars facilitate post-antibiotic expansion of enteric pathogens. Nature. 2013;502(7469):96-99. doi:10.1038/nature12503.

85. Hernandez E, Bargiela R, Diez MS, et al. Functional consequences of microbial shifts in the human gastrointestinal tract linked to antibiotic treatment and obesity. Gut Microbes. 2013;4(4):306315. doi:10.4161/gmic.25321.

86. Song HJ, Shim KN, Jung SA, et al. Antibiotic-associated diarrhea: candidate organisms other than Clostridium difficile. Korean J Intern Med. 2008;23(1):9-15.

87. Vincent JL. Nosocomial infections in adult intensive-care units. Lancet. 2003;361(9374):2068-2077. doi:10.1016/s01406736(03)13644-6.

88. Rice LB. Federal funding for the study of antimicrobial resistance in nosocomial pathogens: no ESKAPE. J Infect Dis. 2008;197(8):10791081.doi:10.1086/533452.

89. Card RM, Warburton PJ, MacLaren N, Mullany P, Allan E, Anjum MF. Application of microarray and functional-based screening methods for the detection of antimicrobial resistance genes in the microbiomes of healthy humans. PLoS One. 2014;9(1):e86428. doi:10.1371/journal.pone.0086428.

90. Field W, Hershberg R. Alarmingly high segregation frequencies of quinolone resistance alleles within human and animal microbiomes are not explained by direct clinical antibiotic exposure. Genome Biol Evol. 2015;7(6):1743-1757. doi:10.1093/ gbe/evv102.

91. Forslund K, Sunagawa S, Kultima JR, et al. Country-specific antibiotic use practices impact the human gut resistome. Genome Res. 2013;23(7):1163-1169. doi:10.1101/gr.155465.113.

92. Hu Y, Yang X, Qin J, et al. Metagenome-wide analysis of antibiotic resistance genes in a large cohort of human gut microbiota. Nat Commun. 2013;4:2151. doi:10.1038/ncomms3151.

93. de Vries LE, Valles $Y$, Agerso $Y$, et al. The gut as reservoir of antibiotic resistance: microbial diversity of tetracycline resistance in mother and infant. PLoS One. 2011;6(6):e21644. doi:10.1371/ journal.pone.0021644.
94. Sommer MOA, Dantas G, Church GM. Functional characterization of the antibiotic resistance reservoir in the human microflora. Science. 2009;325(5944):1128-1131. doi:10.1126/ science.1176950.

95. Buelow E, Gonzalez TB, Versluis D, et al. Effects of selective digestive decontamination (SDD) on the gut resistome. J Antimicrob Chemother. 2014;69(8):2215-2223. doi:10.1093/jac/dku092.

96. Perez-Cobas AE, Artacho A, Knecht H, et al. Differential effects of antibiotic therapy on the structure and function of human gut microbiota. PLoS One. 2013;8(11):e80201. doi:10.1371/journal. pone.0080201.

97. Ghosh TS, Gupta SS, Nair GB, Mande SS. In silico analysis of antibiotic resistance genes in the gut microflora of individuals from diverse geographies and age-groups. PLoS One. 2013;8(12):e83823. doi:10.1371/journal.pone.0083823.

98. Shoemaker NB, Vlamakis H, Hayes K, Salyers AA. Evidence for extensive resistance gene transfer among Bacteroides spp. and among Bacteroides and other genera in the human colon. Appl Environ Microbiol. 2001;67(2):561-568. doi:10.1128/ aem.67.2.561-568.2001.

99. Bahl MI, Sorensen SJ, Hansen LH, Licht TR. Effect of tetracycline on transfer and establishment of the tetracycline-inducible conjugative transposon Tn916 in the guts of gnotobiotic rats. Appl Environ Microbiol. 2004;70(2):758-764. doi:10.1128/ AEM.70.2.758-764.2004.

100. Alpert CA, Mater DD, Muller MC, Ouriet MF, Duval-Iflah Y, Corthier G. Worst-case scenarios for horizontal gene transfer from Lactococcus lactis carrying heterologous genes to Enterococcus faecalis in the digestive tract of gnotobiotic mice. Environ Biosafety Res. 2003;2(3):173-180. doi:10.1051/ebr:2003010.

101. Whittle G, Shoemaker NB, Salyers AA. The role of Bacteroides conjugative transposons in the dissemination of antibiotic resistance genes. Cell Mol Life Sci. 2002;59(12):2044-2054.

102. Furuya EY, Lowy FD. Antimicrobial-resistant bacteria in the community setting. Nat Rev Microbiol. 2006;4(1):36-45. doi:10.1038/nrmicro1325.

103. Nordgard L, Brusetti L, Raddadi N, Traavik T, Averhoff B, Nielsen KM. An investigation of horizontal transfer of feed introduced DNA to the aerobic microbiota of the gastrointestinal tract of rats. BMC Res Notes. 2012;5:170. doi:10.1186/1756-0500-5-170.

104. Aagaard K, Petrosino J, Keitel W, et al. The Human Microbiome Project strategy for comprehensive sampling of the human microbiome and why it matters. FASEB J. 2013;27(3):1012-1022. doi:10.1096/fj.12-220806. 